\title{
Asymmetries in Representational Drawing: Alternatives to a Laterality Account
}

\author{
Jyotsna Vaid $^{1}$
}

\begin{abstract}
When drawing familiar objects there is a bias in starting location, stroke direction, and object orientation or facing. Directional biases are also apparent in the speed and accuracy with which rightward vs. leftward facing objects are recognized and in aesthetic preference. Two different explanatory principles have been offered for directionality effects, one based on attentional/representational asymmetries arising from cerebral hemispheric specialization, and the other based on motoric factors influenced by biomechanical and/or cultural variables. These two accounts lead to differing predictions about the nature and strength of directionality effects in right vs. left-handed users and in users of left-to-right vs. right-to-left scripts. The available evidence suggests that a motoric rather than a laterality account is a more parsimonious explanation of directionality effects.
\end{abstract}

\section{Introduction}

"An unpremeditated profile drawing, if done by a right-handed draftsman, will be represented looking to the left as, if it is the work of a left-handed draftsman, it will certainly look to the right" (Wilson, 1885, cited in Alter, 1989).

"S[ubject]s from other cultures who read and write from right to left should be included in the study ... before final interpretations are justifiable." (Ross, 1951, cited in Jensen, 1951, p. 80).

1 In the interest of space and because there are other interpretive issues arising from the normal developmental and clinical neuropsychological literature on drawing directionality, the focus of this review is on studies of directionality in normal adults. Further, I have chosen to focus predominantly on representational drawings, rather than on the many studies of symbol copying or geometric drawings.

I am grateful to Hsin Chin Chen, Chaitra Rao, and Rebecca Rhodes for assistance in the preparation of this manuscript. 
As ancient cave paintings of horses, lions, and human figures attest, representational drawing is an activity that humans have engaged in for at least 30,000 years. An apparently universal human activity and one that emerges early in life, drawing has attracted the interest of researchers from a variety of perspectives, including developmental psychology, motor perception, and cognitive neuroscience (e.g., Trojano, Grossi, \& Flash, 2009). Representations of common figures typically show directionality effects in perception, production, preference, and recognition. The focus of the present review is on mechanisms underlying asymmetries in the processing of depictions of objects as a function of semantic category (e.g., animate/inanimate, stationary/moving, graspable/not-graspable) and execution parameters (starting location, stroke sequencing, facing).

There are differing views as to the source of directionality effects in object perception and production (e.g., Chokron, 2002). A prevailing view, particularly among neuropsychologists, is that directionality effects arise from representational and/or attentional asymmetries in cerebral hemispheric functioning. According to this view, which we will call the laterality account, directional tendencies are predominantly biological in origin. An alternative to the laterality account regards asymmetries in drawing preference and production as arising from directional biases associated with reading and writing direction. In this view, which we will call the script directionality account, individuals favor a particular side of space or direction of movement depending on their reading/writing direction. A third view, which we will call the biomechanical or chiral account, maintains that directionality effects reflect neuromuscular principles affecting the ease of execution of inward vs. outward-directed hand or limb movements. In this view, biomechanical constraints arising from the anatomical structure of the hands, arms and nervous system are thought to influence the preferred direction of stroke production by adults (see Van Sommers, 1984). A fourth view, the chi$\mathrm{ral} /$ scriptal account, suggests that reading/writing habits constitute a form of motoric influence and interact with biomechanical variables to affect drawing directionality.

In reviewing the available studies of drawing directionality, we will conclude that a chiral/scriptal view appears to be able to account for more of the findings than the other views.

\section{Hemisphere/attentional accounts of asymmetries in perception and/or production}

Numerous studies with neurologically intact individuals conducted since the 1960s have shown that when words are briefly presented to the right visual 
half field participants are more accurate in identifying them than when the same items are presented to the left visual half field. This right visual field superiority was interpreted as support for a left cerebral hemisphere representational and/or attentional bias for language (Kinsbourne, 1970).

However, as Bryden and Mondor (1991, p. 428) pointed out in a review of the laterality literature, "[d]espite this predilection for interpreting visual field effects in terms of functional cerebral asymmetries, there have been regular cautionary reminders that the procedure may not be so simple to interpret." Among the numerous factors besides cerebral specialization that also appear to influence the magnitude and direction of visual field asymmetries is that of scanning biases arising from experience in reading or writing from left to right (see, e.g., Nazir, Ben-Boutayab, Decoppet, Deutsch, $\&$ Frost, 2004). In acknowledging that scanning effects offer a potential alternative account of visual field asymmetries one is not necessarily arguing that visual field asymmetries in perception or production have nothing to do with cerebral functional asymmetries, but simply that it is incumbent on researchers to consider whether asymmetries can be accommodated more parsimoniously in other ways. In what follows we evaluate laterality vs. non-laterality accounts of asymmetries in directionality.

Heron (1957) noted that there are two kinds of scanning effects associated with reading and writing: one involves scanning to the beginning of a text (i.e., to the left in left-to-right readers) and the other involves scanning from one word to the next in a line of text (i.e., from left to right in English readers). Thus, under bilateral viewing conditions readers of English would be expected to orient their attention first to the left side of space whereas under unilateral presentation conditions they would more easily scan from the center to the right side of space, and thereby show a right field advantage in word recognition. A right field superiority in these participants, therefore, need not relate to hemispheric functional asymmetry at all.

Proponents of a laterality account have acknowledged the possibility of a scanning artifact but have tended to discount it. Attempts to test for scanning biases in laterality studies by using readers of Hebrew, a language ostensibly written from right to left, have been inconclusive. However, the lack of definitive findings from studies of Hebrew readers may in part be due to the fact that typical participants in these studies also know English and thus are more properly characterized as bidirectional readers. Further, Hebrew itself may be considered bidirectional given that individual letters, numbers and musical notes are written from left to right although words are read from right to left.

Fortunately, some studies have been conducted with readers of other languages, such as Urdu, that are more consistently right to left in their script directionality. Vaid, Rao and Chen (2009) compared visual field asymme- 
tries for Urdu vs. Hindi among readers of both scripts in north India. These languages are identical in phonology and grammar but differ primarily in orthography and script directionality. In Hindi word recognition, participants showed a strong right visual field advantage; when tested in Urdu, a comparable right visual field advantage was obtained (see also Vaid, 1988, who reported an equivalent right visual field asymmetry in native Hindi and native Urdu readers, and Adamson \& Hellige, 2006, who reported a right field advantage in Urdu-English skilled readers). These findings suggest that a laterality account may indeed be a viable explanation of observed asymmetries in word recognition since even readers of right-to-left scripts show a right visual field superiority.

However, if a laterality account may serve to explain visual field asymmetries in verbal processing in left-to-right and right-to-left readers alike, it remains to be determined if it also provides a sufficient account of visual field asymmetries in nonverbal tasks or whether reading/writing habits affect performance on such tasks and offer an alternative explanation to a right hemisphere/left hemispatial bias account.

To address this issue let us consider studies bearing on two ostensibly nonlinguistic tasks - facial affect judgments and aesthetic preferences both of which have been mainly interpreted within a laterality framework.

\subsection{Face perception directionality}

In the free viewing version of the chimeric faces task (Levy, Heller, Banich, $\&$ Burton, 1983), participants are shown pairs of chimeric faces in which either the left or the right half of the face from the viewer's perspective is smiling. On this task a left field bias is typically observed; that is, participants favor the face in which the smile is in the viewer's left field as the more expressive, "happier" face. This effect has been replicated across several studies. It is noteworthy that all of the replications of the chimeric faces effect by Levy and her colleagues were done on users of left to right languages. The standard interpretation of the left field bias has been in terms of right hemisphere specialization for facial affect judgments.

Vaid and Singh (1989) administered the chimeric faces task to adult readers of Hindi, Urdu-Hindi biliterates, and illiterate speakers of Urdu/Hindi and found a significant left field bias only in the Hindi readers, that is, the left-to-right readers. Three subsequent studies have replicated and extended Vaid and Singh's (1989) finding. Sakhuja, Gupta, Singh and Vaid (1996) found that the left field bias was reduced in Urdu as compared to Hindi right handers as well as in left handed readers in each group. Eviatar (1997) found a reduced left field bias in native Hebrew readers as compared to native Eng- 
lish readers. Heath, Rouhana and Ghanem (2005), who administered the chimeric faces task to a large sample of Arabic readers in Lebanon using photographed faces from the local population, showed that a left side bias was strongest in the subgroups that had the most exposure to English. Indeed, the association between right-to-left reading/writing experience and a reduced left field preference on the chimeric faces task was confirmed by Eviatar (1997) in a meta-analysis of chimeric faces studies, supporting the script directionality view over the laterality view of the left bias.

A scanning interpretation is consistent with findings from three recent studies, all with left-to-right readers. One found that participants initially look to the left when viewing centrally presented (non-chimeric) faces (Hsaio \& Cottrell, 2009). Another noted that, when viewing a face, viewers first look to the side of space that is contralateral to their dominant eye; thus, right eye-dominant individuals initially look to the left side of a face (Hernandez, Metzger, Magne, Bonnet-Brilhault, Roux, Barthelemy, \& Martineau, 2009). A third study showed a left attentional bias when viewing scenes, with better spatial memory for objects on the left side (Dickinson \& Intraub, 2009). Of course, in the absence of data from right to left readers on these tasks one cannot definitively conclude that a script direction effect underlies the observed biases noted in these studies; however, given that studies of chimeric face viewing with right to left readers showed opposite effects to those of left to right readers (Eviatar, 1997), one can predict that right-to-left readers would show a right field initial gaze preference in viewing centrally presented non-chimeric faces as well.

\subsection{Aesthetic response as a function of directionality}

Another domain in which a right hemisphere account has been prevalent is in studies of aesthetic response to rightward vs. leftward facing photographs, drawings or portraits (e.g., Levy, 1976; McLaughlin, 1986; McLaughlin \& Murphy, 1992). In these studies, a typical effect is that viewers prefer rightfacing figures over left-facing ones, and/or figures in which there is some focal element on the right side of the painting rather than on the left side. It is not clear if this effect is moderated by the viewer's (or the artist's) handedness. Very few studies of aesthetic preference have included sufficient numbers of left handers to allow definitive conclusions about how this variable may affect performance but even where left handers have been included in studies of aesthetic judgments, the results have been inconclusive, (e.g., Mead \& McLaughlin, 1992).

Turning to studies of aesthetic preference, one study examined aesthetic response to spatial placement of mirror-reversed objects shown in a rectan- 
gular frame (Palmer, Gardner, \& Wickens, 2008). Viewers preferred rightfacing objects over their left-facing counterparts; they also preferred objects that faced towards the center rather than towards the edge of the frame (Palmer et al., 2008). Importantly, these same preferences were also found under unconstrained response conditions: three objects - a steam iron, a tape dispenser, and a teapot - were individually placed on a turntable and participants were allowed to rotate the object to whatever orientation they preferred before photographing it. Over $80 \%$ of the participants rotated the objects to face rightward (Palmer et al., 2008, Exp. 4).

A number of studies of aesthetic response have examined the effect of implied direction of movement in a painting. Implied movement in Western works of art was commented on by early art theorists, such as Gaffron (1950), who proposed that viewers enter a painting by scanning it from the lower left and proceeding in an upward rightward arc. Asymmetry in paintings, these early theorists suggested, lends an aesthetic dimension of movement in graphic art; the particular preference for an asymmetry to be located on the right side may in turn reflect the fact that " $[\mathrm{m}]$ ovement from left to right in a painting is easier and faster while movement from right to left is slower and perceived as having to overcome resistance" (Gross \& Bernstein, 1978, p 36).

Freimuth and Wapner (1979) reported that participants preferred paintings in which the implied motion was from left to right rather than from right to left. Banich, Heller and Levy (1989) reported that paintings in which the salient element was located on the viewer's right side and where the implied movement was thus from right to center were preferred (by right-handers only) over those with a left-biased salient element. Banich et al. proposed that having the more important figure on the right side offsets a right-hemisphere-mediated left hemispace attentional bias and creates balance, which is judged pleasing. However, Beaumont (1985) enlisted eye movement data to support his claim that in making aesthetic judgments participants' gaze is first drawn to the left hemispace and then moves rightward towards the asymmetric dimension of interest (see also Mead \& McLaughlin, 1992).

If, as Gaffron (cited in Gross \& Bernstein, 1978, p. 36) noted, "we 'read' a picture in a certain way just as we read a page of a book," one may well ask whether those who read from right to left would show a leftward preference in their aesthetic response. Whereas Gross and Bernstein provide anecdotal examples in support of this idea, four empirical studies bear on this issue.

Chokron and de Agostini (2000) tested 81 right-handed, monolingual readers of French (including 41 Grade 3 children and 40 adults) and 81 righthanded readers of Hebrew from Israel (including 40 Grade 3 children and 
41 adults). Stimuli were 30 pairs of mirror reversed line drawings of objects presented one above the other; participants were asked to judge which member of the pair was more aesthetically pleasing or interesting. The stimulus pairs included ten static (e.g., a statue) and ten mobile, directional objects (e.g., a truck) in which one member of the pair faced leftward and the other faced rightward. In addition, ten pairs of asymmetric landscape pictures were presented in which the salient element was located on the right or on the left side. Chokron and de Agostini (2000) reported that left to right readers preferred pictures that faced rightward, whereas right to left readers preferred pictures that faced leftward. They concluded that these results support a nonlaterality, reading-habit-based account of aesthetic preference, since, " $[\mathrm{t}]$ here is neither experimental nor clinical argument for a reverse pattern of cerebral lateralization in subjects with opposite reading habits" (Chokron \& de Agostini, 2000, p. 48)

However, given the fact that landscapes showed a tendency (significant in the Israeli adults) for a right-side preference, they suggested that a hemisphere-based interpretation might also be at work.

Christman and Rally (2000), cited in Heath, Mahmasanni, Rouhanna and Nassif (2005) tested aesthetic preferences in a sample of left-to-right (English) readers, biliterate (left to right and right-to-left) readers of Arabic and English, and top-to-bottom readers (of Japanese), using geometric stimuli differing in direction of implied movement, weight and interest. They reported a significantly stronger right-movement-directed preference in the left to right group compared to the other two groups. Further, degree of rightward preference in the biliterate and top-bottom readers was correlated with years of exposure to English.

Heath, Mahmasanni, Rouhana and Nassif (2005) also examined aesthetic preferences using geometric stimuli. Their participants included 65 English readers tested in the U.S., 58 native Arabic-only readers, 326 biliterate readers of Arabic and English, and 118 illiterate adults who were speakers of Arabic. All except the first group were tested in Lebanon. Participants ranged in age from 17 to 75 . The test items, presented in booklet form, consisted of 44 different permutations of three geometric elements configured to represent four levels each of weight and interest (right, on left, balanced, or absent), and two levels of implied movement direction (left to right or right to left). Mirror images of the stimulus arrays were presented in pairs placed one above the other, and participants were to indicate which array was more appealing. As only the 13 pairs that contained movement directionality cues showed a significant bias, analyses were done only on those. A significant group difference interacting with the other factors showed that English use was associated with more rightward preference; further, in the English-only participants, a rightward movement bias was 
enhanced when the "interest" element was placed to the left rather than to the right. By contrast, the Arabic- only readers showed a leftward movement bias when the interest element was placed to the right. Biliterates and illiterates did not show interpretable effects. It is difficult to know how participants were actually evaluating the fairly abstract geometric stimuli used in this study and, in particular, whether the experimenter-designated labeling of the elements conveying interest, weight or directionality in fact matched participants own perceptions of the stimuli. Nevertheless, script direction appears to have differentially influenced preference judgments.

Whereas the studies of aesthetic responses of right to left readers reviewed thus far looked only at right handers, one study reviewed below included left handers as well. Nachson, Argaman and Luria (1999) presented participants with left-facing and right-facing profiles of human faces and bodies taken from art books. Stimuli were presented side by side in mirror image pairs with the members facing outward or inward. Participants were normal adult readers of Arabic (28 right-handed, 17 left-handed), Hebrew (54 R, 8L), and Russian (16R, 15L), all tested in Israel. They were to indicate whether the left or the right member of the pair was the more beautiful. It was found that Russian readers preferred right-directed profiles whereas Hebrew readers showed a significant preference for left-directed profiles; Arabic readers' preferences, though showing a tendency for a left-directed preference, did not differ from chance. A handedness by stimulus direction interaction indicated that, across groups, right handers preferred leftdirected profiles, whether in outward- or inward-directed pairs of faces or bodies, whereas left handers showed a right-directed preference for all conditions except for inward-directed facial profiles. A near significant handedness by language group interaction suggested that differences between right and left handers were most pronounced in the Russian readers, with Russian left handers showing the strongest right-directed preference. The authors concluded that "stimuli with directional dynamics (such as profiles) that correspond to the participant's direction of scan are judged as being more beautiful than those with directional dynamics that oppose the direction of scan, thus demonstrating a link between aesthetic preference and reading/writing habits" (Nachshon et al., 1999, pp. 111-112). This study clearly suggests that reading/writing habits influence aesthetic judgments; however, replications of this study in which the stimuli are presented one above the other rather than side by side would strengthen the argument.

Taken together, we have seen that in the case of two nonlinguistic domains - facial affect judgments and aesthetic judgments - the predominant outcome of a left hemispatial bias in the former case and a right facing bias in the latter was restricted to readers of scripts that proceed from left to right. In users of right to left or bidirectional scripts the effect was much 
weaker or even reversed, suggesting that reading/writing habits provide a sufficient alternative account of directional asymmetries in perceptual tasks without the need for invoking laterality differences.

\subsection{Directional asymmetries in drawing directionality}

Directionality in drawing may arise at planning or execution stages of drawing and may be revealed in a tendency to start a drawing on a particular quadrant of the page, on a particular side of the object to be drawn, in stroke direction, and/or in the orientation or facing of the object (Van Sommers, 1984). For each of these parameters a clear asymmetry has been observed. Aymmetries have also been observed in the identification of objects that face left vs. right (Viggiano \& Vannucci, 2002), and in the accuracy of recall or recognition of right- vs. left-facing objects (Martin \& Jones, 1999).

\subsection{Accounts of the origin of directionality effects in drawing}

There is a longstanding association between damage to the right hemisphere and visual neglect of the left side of space (see Bartolomeo, 2007, for a review). When drawing common objects from memory or when copying drawings of objects, neglect patients tend to omit left side details. The availability of visual feedback appears to exacerbate left neglect, as drawing with eyes closed results in more complete drawings in left neglect patients (Chokron, Colliot, \& Bartolomeo, 2004). That drawing recruits more right hemisphere regions was suggested by a recent fMRI study of neurologically intact individuals which reported a stronger activation of a region in the right frontal cortex (BA 44/45) when participants were asked to imagine drawing as compared to a condition where they were to imagine writing (Farias et al., 2006, Exp. 2). Findings such as these support the view that processes involved in spatial attention and object perception more generally are associated with right hemisphere functioning.

Drawing orientation or the right- vs. left-facing of objects with an intrinsic front appears to vary by handedness. Some researchers have wondered whether this association of drawing directionality with handedness may signal that drawing directionality is lateralized. Thus, for example, Levy and Reid (1978) included a profile drawing task in a series of laterality tests administered to right- and left-handed adults "to see if profile orientation might prove an additional behavioral measure indicating cerebral dominance" (p. 126). Alter (1989, p. 563) administered a drawing task to a large sample of individuals varying in handedness and proposed a way of quanti- 
fying the assessment of drawing directionality and relating it to measures of cerebral laterality, noting that "[t]he case will be made that 'directionality' or the tendency to a stable perceptuo-motor bias - is cerebrally lateralized." Alter claimed that the tendency for leftward facing, which is especially pronounced in right handers, was associated with a stronger right visual field preference on verbal laterality tasks (Alter, Rein, \& Toro, 1989).

A rather different conceptualization of handedness was proposed by Martin and Jones (1999), who proposed a chiral account whereby handedness is seen as exerting a direct, motoric effect in the recognition and production of objects facing rightward vs. leftward. Right and left handers, in this view, differ in their performance on a range of cognitive tasks not because of differences in cerebral lateralization but because of differences in motoric activation associated with left vs. right hand use. For example, it is well established that tensor movements, or movements directed away from the body midline, are performed more smoothly and rapidly than flexor movements, or movements directed towards the body (Brown, Knauft \& Rosenbaum, 1948). This would lead to different outcomes in drawing directionality depending on whether the right or the left hand is used to draw.

\section{Different predictions of biomechanica/chiral and laterality accounts of drawing directionality}

According to the biomechanical account, right handers using their dominant hand should be more likely to draw profiles facing left whereas left handers using their dominant hand should draw profiles facing right, simply because of the greater motoric ease in each case of making outward rather than inward movements. By the same token, right handers should be better at remembering left-facing than right-facing objects, and should misremember right-facing objects as left-facing, reflecting a left-looking memory schema. A biomechanical/chiral account would also predict that the way one normally interacts manually with an object should affect its ease of identification as well as how it is depicted in drawing. Thus, manipulable objects should be drawn or pictured oriented in a way that reflects usage - so, for example, handles of saucepans should be aligned to the same side of space as the hand used to draw them (i.e., to the right side in right handers and to the left in left handers).

To the extent that handedness in the laterality account is seen as an indirect marker of lateralization, directionality effects observed in left handers should be in the same direction, but not as strong in their magnitude, as those observed in right handers. By contrast, the biomechanical/chiral view would predict that right and left handers should show equally strong but 
opposite directionality effects, reflecting the different neuromuscular factors underlying graphic production depending on hand used. Furthermore, whereas the variable of handedness (or preferred hand) is emphasized in the laterality account, the biomechanical account emphasizes hand used, regardless of hand dominance, as the variable of interest (e.g., Braswell \& Rosengren, 2002).

Finally, a chiral view can, in principle, accommodate differences associated with writing habits or script directionality. It would predict that directionality effects represent an interaction of biomechanical and cultural influences on hand movement biases. Thus, when script direction is congruent with hand movement-related directional preferences directionality effects should be strong (as when left to right readers perform a drawing task with their right hand or right to left readers perform the task with their left hand) Directionality effects should be weaker when biomechanical variables counter script directionality effects, as when left to right readers perform a drawing task with their left hand (script directionality would favor left to right movement whereas biomechanical variables would favor right to left movements) or when right to left readers perform a drawing task using their right hand (a right to left script-based movement preference would go against a left to right biomechanical movement preference). The relative strength of script direction vs. biomechanical variables in any given task becomes an empirical issue. A laterality account is silent on the issue of script direction and its potential interaction with hand used.

\section{Drawing directionality effects in right- vs. left handed users of left to right scripts}

We now review findings that bear on these predictions, with particular attention to studies of the drawing of horizontal lines, human profiles, animals, and objects that convey movement (e.g., vehicles), and/or are graspable (e.g., have handles).

\subsection{Drawing Horizontal Lines}

The biomechanical principle of tensor movements being executed more easily than flexor movements would lead to a prediction that right handers will tend to draw horizontal lines from left to right when using their dominant hand, whereas left handers will draw them from right to left when using their dominant hand. Scheirs (1990) compared right-handed and lefthanded 4-to12-year old children on a series of actions including using 
a hammer, drawing circles, tracing a circle in the air, boring into wood with a tool, drawing a human profile, and drawing horizontal lines. He found that the latter task was the only one on which consistent differences were observed between right and left handers.

Braswell and Rosengren (2002) tested 67 4-6 year olds and 15 adult right handers on a figure copying task to be performed repeatedly over six trials by each hand. On the figure with horizontal components (a cross), they found opposite movements in the adults as a function of hand used: when using their right hand, participants mainly produced horizontal lines in a left to right direction whereas when using their left hand, they mainly produced the lines in a right to left direction.

Several other studies have replicated this effect in comparisons of rightvs. left handed children and adults: right handers consistently produce lines in a left to right direction whereas left handers do so from right to left (see de Agostini \& Chokron, 2002; Dreman, 1974; Glenn, 1995; Lehman \& Goodnow, 1975; Reed \& Smith, 1961; Shanon, 1979; Von Sommers, 1984). The size of the effect is equally strong in right and left handers, supporting the predictions of the motoric view.

A similar outcome is found on other graphic production tasks involving horizontal movements, such as when participants have to rapidly place dots in a horizontal array of boxes; performance by right handers is significantly faster when proceeding in a left to right direction whereas that by left handers is significantly faster in a right to left direction (Vaid, 1998). Singh, Vaid and Sakhuja (2000) found that lines drawn to approximate a target line by right handed left to right readers of Hindi were closer to the target in actual length when drawn from left to right, whereas lines drawn by individuals with bidirectional writing experience (Hindi/Urdu) were equally accurate whether drawn from left to right or from right to left.

\subsection{Drawing human facial profiles}

Painted portraits tend to look towards the observer's left (Humprey \& McManus, 1973), as do school yearbook pictures, and photographs of celebrities. This tendency for leftward orientation of human faces is also consistently observed when people - particularly right-handers- are asked to draw profiles of faces (see Richardson, 1992; Van Sommers, 1984, pp. 6-8, 120-122). One of the earliest and most extensive studies of profile drawing directionality was that by Jensen (1952) who examined this effect in a number of different groups. Jensen found that $64 \%$ of a sample of 355 American right handed children showed a left facing bias as compared to $42 \%$ of a much smaller sample of left handed children $(n=33)$. A left bias 
was also observed in $84 \%$ of right handed American college students $(\mathrm{n}=88)$, and $88 \%$ of right-handed art students $(\mathrm{n}=16)$, and was replicated in a sample of Norwegians (Jensen, 1952).

Crovitz (1962) investigated the relationship between handedness and profile drawing directionality in a group of 375 American college students. Like Jensen, he found a left facing bias among right handers, which was significantly different from that of left handers. Levy and Reid (1978) examined profile drawing orientation in 24 right-handed and 48 left- handed adults, the latter further subdivided as a function of hand posture while writing (inverted vs. non-inverted). The vast majority (83.3\%) of right handers showed a left-facing bias, as compared to $33.3 \%$ of the non-inverted lefthanders and $25 \%$ of the inverted left handers. Shanon (1979) similarly reported a left facing preference for human profiles in $77.5 \%$ of right handed adults $(\mathrm{n}=40)$ as compared to $47.5 \%$ of left handers $(\mathrm{n}=40)$.

Vaid (1995) reported a left facing bias in profile drawing among $89.1 \%$ of Hindi right handers $(n=55)$. Martin and Jones (1999) reported a left facing bias for human profiles in $57.4 \%$ of right handed adults $(n=166)$ as compared to $38.5 \%$ of left handers ( $\mathrm{n}=110)$. De Agostini and Chokron (2002) reported that in young adults a left facing bias was evident in $75 \%$ of right handers vs. $50 \%$ of left handers $(n=20$, each); among older adults, a left facing bias was evident in $89 \%$ of right handers $(n=37)$ but only $20 \%$ of left handers $(n=5)$. Karev (1990) found that $92.4 \%$ of right handers $(n=264)$ showed a left-facing bias, as compared to $70.7 \%$ of left handers $(n=270)$.

\subsection{Drawing animals, vehicles, and objects}

Vaid (1995, Study 1) reported a strong left bias in the facing of bicycles $(90.9 \%)$ and elephants $(92.7 \%)$ in a sample of 55 right-handed Hindi speaking adults. In a follow-up study investigating 16 right- and 16 left-handed Hindi users between the ages of 9-13 who were asked to draw a fish, an arrow, and a flag, right handers were significantly more left-facing than left handers (Vaid, 1995).

Alter (1989) tested 212 right handers and 42 left handers ranging in age from 11 to 72 years in a drawing task involving six objects: a bicycle, a dog walking, a bus, a facial profile, an airplane, and a pitcher. Data were not presented separately per drawing. Alter (1989) found that right handers were significantly left-directed whereas left handers were right-directed in their drawings. Importantly, right-and left handers did not differ in their degree of directional bias. Further, of those who oriented the drawings to the left, the vast majority (83\%) were right-handed (only 3\% were left- 
handed); among right-directed participants, handedness was evenly represented across right, left and mixed handers.

Using the same items as Alter (1989), Karev (1999) tested 264 right handed adults and 270 left handers, as well as a sample of mixed handers. Left-facing directionality was observed across groups but was significantly greater in right handers. Further, the frequency of right-directed drawings was greatest in left handers.

\section{Animacy}

Vaid and Chen (2009, Exp. 1) examined differences in drawing directionality in a sample of 284 right handed and 145 left handed native English speakers on the following items: fish, arrow, profile, elephant, bicycle, and flag. Handedness differences were observed for all three animate items profile, fish, and elephant - in the direction of a greater left facing bias among the right handers. There were no handedness differences for the inanimate items (arrow, bicycle, flag). Martin and Jones (1999) similarly reported significant handedness difference in drawing directionality for profiles but not for the drawing of bicycles. However, the apparent conclusion from these two studies that directionality differences between right and left handers are restricted to animate objects is not a consistent finding across other studies.

\section{Movement}

Viggiano and Vannucci (2002, Exp. 1) administered a drawing test to 115 right handed and 75 left handed participants between the ages of 18 and 30. Participants were to draw 60 animals, 31 vegetables and 155 nonliving objects (vehicles, tools, furniture) with their preferred hand in two separate sessions. Percent frontward, leftward, and rightward facing was analyzed. No handedness or orientation differences were observed for vegetables, tools, or furniture. However, significant differences were obtained in the facing of animals and vehicles: $71 \%$ of right handers drew animals facing leftward while $65 \%$ of left handers showed a rightward orientation. For vehicles, $58 \%$ of right handers oriented them to the left whereas $66 \%$ of left-handers oriented them to the right.

\section{Object graspability}

Karev (1999) reported that the facing of a jug depended on participants' handedness: there was a clear preference for drawing the handle in the same side of space as the dominant hand: i.e., to the right in the case of 
right handers and to the left in the case of left handers. A similar effect was noted by Vaid and Chen (2009) in the drawing of a teacup. These findings demonstrate that graspable objects are drawn in the direction of the hand with which they are likely to be grasped.

de'Sperati and Stucchi (1997) showed 15 right handers and 15 left handers between the ages of 20 and 46 years a computer animation of a screwdriver in different orientations moving in a righward or leftward motion. Participants were asked to judge the direction of motion. In another condition, they were to imagine holding the screwdriver with either their dominant or nondominant hand and then decide on the motion. Participants' ability to recognize the motion of the screwdriver was affected by its orientation, such that the farther away the handle was positioned from the observer, the longer the response latency. Only right handers showed a preference for the screwdriver orientation in which the handle was to the right. In the imagined holding condition, both right and left handers imagining holding the screwdriver with the right hand showed a preference for rightward orientations whereas there was no orientation preference for imagined left hand holding. These results were taken as support for the spontaneous use of motor imagery for internally simulating movements of the preferred hand resulting in different response times depending on the graspability of the visual stimulus (see also Symes, Ellis \& Tucker, 2007).

\section{Dominant vs. non-dominant hand used}

Five studies of left to right readers examined the effects of drawing with the dominant vs. the non-dominant hand. The earliest study to examine this variable was that of Crovitz (1962), which proposed that differences in drawing orientation "may be related to a simple peripheral sensory-motor variable; viz., whether the pencil is held in the left vs. the right hand" (Crovitz, 1962, p. 196). Eleven right handers were asked to draw a profile using their right hand, and 11 other right handers did so using their left hand. Crovitz found that those using the right hand drew significantly more left-facing than right-facing profiles, whereas those using the left hand drew more right-facing than left-facing ones.

Examination of the detailed movements made in drawing a figure may clarify why the drawings end up facing as they do. The relative ease of drawing a left-facing figure when using the right vs. the left hand, Crovitz (1962) suggested, may relate to different movement patterns; in the right hand, "[t]he elbow is close to the body, the ulnar surface of the hand is in contact with the writing surface, the hand is in line with the arm, the pencil point is on an extension of this line, and the first pencil stroke is laid down by a wrist flexion. This counterclockwise pencil stroke lays down the fore- 
head of a left-facing figure. When the pencil is held in the left hand, precisely the same movement pattern lays down the forehead of a right-facing figure" (Crovitz, 1962, p. 196).

An additional variable here may be that when using the right hand lateral movements are easier to make in a left to right direction. Taguchi and Noma (2005) studied 20 right-handed Japanese adults on a fish drawing and circle drawing task performed with each hand. They found an overall left facing bias which was stronger when the right hand was used; further, a relationship was found between left-facing of the fish and the use of a clockwise movement in circle drawing, but only when the right hand was used. Thus, tendency to draw circles in a clockwise movement (which involves a left to right movement direction) was correlated with a tendency to face fish to the left, suggesting that the fish were drawn from left to right as well, starting with the head (see also van Sommers, 1984).

Alter (1989) asked right- and left-handed participants to draw one of six objects using their non-dominant hand as well as their dominant hand. She found that switching hands generally did not change drawing orientation; in the few instances where it did, this was more likely for left-handers than right handers. Given that the actual object to be drawn with each hand differed across participants, it is hard to know how to interpret Alter's (1989) findings on the effects of hand used on drawing directionality. Nevertheless, it is noteworthy that where drawing orientation shifted by hand used, this was more likely to be the case for left handers than right handers.

Vaid and Chen (2009, Exp. 2) tested 161 right handers and 64 left handers on a profile drawing task with each hand, with hand order counterbalanced. They found a main effect of hand order which indicated a stronger overall leftward facing bias when the dominant hand was used first. A main effect of hand used was also found, as was a hand used by handedness interaction. The interaction indicated that right handers showed the same pattern of facing whether they used their right or their left hand; left handers, by contrast, showed a stronger leftward facing bias when using their right hand than when using their left hand. In other words, the direction of writing was a stronger influence than biomechanical factors for right handers.

\subsection{Identifying objects}

Viggiano and Vannucci (2002, Exp. 2) hypothesized that right and left handers should show differences in the ease of identification of objects facing leftward vs. rightward, respectively. Participants (37 right handers and 23 left handers) were shown 84 line drawings of objects (40 animals, 8 vehicles, 14 items of furniture, and 22 tools) in fragmented versions at the 
center of a screen for $100 \mathrm{~ms}$ in a sequence of three levels of fragmentation, keeping the direction of facing of the objects across levels.

Viggiano and Vannucci (2002) found a three way interaction of handedness, directionality and object mobility. No handedness differences were observed for immobile objects (furniture, tools); for mobile objects (animals, vehicles) right handers named leftward facing objects faster than rightward facing ones; the converse was found for left handers. Moreover, right handers identified leftward facing animals at lower threshold levels than they identified rightward facing ones, while left handers showed no threshold differences. The authors interpreted their results as supporting the motor imagery theory of Martin and Jones (1999) which proposes an isomorphism between the structure of a movement and the structure of the image or mental representation. According to this theory, one should expect the same pattern of findings from tasks that require the manipulation, identification or recognition of the same visual object, indicating that the mental representation involved in motoric acts such as drawing, and in visual processes such as object identification, contain a description of the directionality of the object.

\subsection{Recognizing object orientation}

Three studies have examined the accuracy of orientation memory for figures. Takala (1951) found that when asked to reproduce an array of geometric figures presented in specific orientations, right handers were better at remembering the position of left-facing than right-facing figures. This effect was also observed by McKelvie (2001), who tested incidental recognition memory for facial orientation in 241 adults across three experiments. $\mathrm{He}$ found that faces that looked to the left elicited better orientation memory accuracy than those that looked to the right, suggesting that people have a schema that faces generally look to the left. Martin and Jones (1999) found that memory for coins, faces of famous figures, and other faces was generally better for leftward facing profiles for right handers but for rightward facing profiles by left handers.

\section{Drawing directionality effects in right- vs. left-handers as a function of script directionality}

\subsection{Developmental studies of drawing directionality}

Many developmental studies have compared readers of left to right scripts (English) with readers of right to left scripts (Hebrew) on symbol copying 
tasks that involve horizontal or circular movements (see Nachshon, 1981). However, as with the studies of perceptual asymmetries, the findings from drawing directionality do not provide a clear picture of script directionality influences. For reasons already noted, Hebrew may not be a particularly good candidate to test claims about directionality effects. When English is introduced in school Israeli school children show a sharp shift in their directionality effects, presumably reflecting the influence of exposure to a left to right script. Importantly, this shift does not occur in children who were taught both Hebrew and English scripts at the same time (Nachshon, 1983). Interestingly, children who learned scripts with opposing directionality from the start show directionality effects that are different from those of either monoliterate group (Nachshon, 1983).

\subsection{Estimating length of lines produced from left to right vs. right to left}

Singh, Vaid, and Sakhuja (2000) compared left- and right-handed schoolchildren with unidirectional left-to-right (Hindi) or bidirectional (Hindi and Urdu) reading/writing experience on a line length judgment task. Participants were asked to draw $3 \mathrm{~cm}$ lines from left to right or from right to left with each hand. Regardless of hand used, lines drawn from left to right were closer in length to the target than those drawn from right to left in right handed unidirectional readers. Bidirectional readers showed no directional effect. Furthermore, bidirectional readers produced lines that were closer in length to the target line than were unidirectional readers. These results support a greater influence of script-related directional scanning effects than handedness on the task of line length estimation.

\subsection{Speeded dot filling of horizontal arrays}

Vaid (1998) tested readers of Arabic vs. English on a task requiring participants to place a dot in a row of boxes from left to right or from right to left as quickly as possible, using the right or the left hand. A biomechanical account would predict faster performance in an outward direction (i.e., left to right with the right hand, right to left with the left hand) than in an inward direction. This was supported except when biomechanical movement patterns conflicted with the preferred (right to left) writing direction of the users, in which case script direction influenced movement speed (Vaid, 1998). 


\subsection{Drawing human facial profiles}

As already noted, the majority of studies of human facial profile drawing directionality that have been conducted with readers of left to right languages have shown a strong leftward facing bias, which is stronger in rightthan in left-handers. With right to left readers one might expect a tendency for a rightward bias. In one of the earliest studies to include a sample of right to left readers, Jensen (1952) reported that, of 90 Grade 3-8 right handed Arabic schoolchildren tested in Egypt, only 34\% drew profiles facing rightward. This effect did not differ from that of right-handed American children. However, Jensen did not include left-handed users of Arabic. Shanon (1979) compared profile drawing in right vs. left handed adult users of Hebrew and found a right-facing tendency in $15 \%$ of the right handers but in $60 \%$ of the left handers ( $n=40$ per group).

Vaid (1995) found a nonsignificant right-facing tendency in $54.8 \%$ of right handed Arabic readers $(\mathrm{n}=82)$ as compared to a significant left-facing bias $(86.8 \%)$ in right handed Urdu-Hindi readers $(n=38)$ and Hindi readers $(89.1 \%, n=55)$; left handers were not tested on this task. Vaid \& Chen (2009, Exp. 3) examined Arabic vs. Hindi right-handers' facing of profiles as a function of hand used. They found a significantly greater right-facing bias in right-handed Arabic readers $(\mathrm{n}=50)$ as compared to Hindi readers $(n=30)$, regardless of whether they used their right or their left hand to draw: Arabic - right hand $=64 \%$, left hand $=66 \%$, Hindi - right hand $=13.3 \%$, left hand $=16.7 \%$.

\subsection{Drawing animals, vehicles and other objects}

Vaid (1995) reported a significant rightward facing of bicycles $(65.9 \%)$ and elephants $(63.3 \%)$ in right-handed Arabic readers $(n=82)$, whereas right handed Hindi readers showed a strong left facing bias for these objects. Vaid \& Chen (2009, Exp. 3) compared drawing orientation in Arabic and Hindi readers as a function of hand used and found that, regardless of hand used, a significantly greater rightward facing bias characterized Arabic vs. Hindi readers in the drawing of an elephant (58\% vs. $16.1 \%)$, a bicycle (68.7\% vs. $16.1 \%)$, a shoe $(62.2 \%$ vs. $8 \%$ ), and a cup $(29.8 \%$ vs. $3.2 \%)$. The variable of hand used was significant only for the item, cup: the handle was placed on the right when the right hand was used to draw; this effect was considerably reduced when the left hand was used to draw. There was a tendency for an interaction of hand used with group, suggesting that Hindi readers were much less likely to show a change in orientation as a func- 
tion of a switch in hands, suggesting that script direction had a greater influence than hand movement bias due to biomechanical variables.

To date, no studies of object naming, object perception, or memory for object orientation have been done in right to left readers.

\section{Concluding remarks}

An overview of existing studies suggests that the available evidence on drawing directionality is more compatible with a motoric account (which includes an influence of script directionality) than with a strictly lateralitybased account (which makes no predictions about opposite effects as a function of hand used, or script direction).

Further research on directionality effects would do well to address certain methodological weaknesses. In all but one study of drawing directionality (Viggiano \& Vannucci, 2002), fewer than 10 stimuli have been used. When only a few items are used, and particularly when the items differ in how they are produced (e.g., for some items, such as a fish or a profile of a face, the directional element tends to be drawn first whereas for others, e.g., the tip of an arrow, or the handle of a teacup, it is drawn last) and thus in how they may end up being faced, it is important to analyze facing orientation separately by object. To reduce variance across studies, it would be important to standardize such aspects of the procedure as whether the drawings are to be made on separate sheets of paper or on a single sheet containing a grid, whether the paper is positioned upright or allowed to be tilted to the right or left, whether the drawing is to be made within a frame, and whether the frame is symmetrical (e.g., square or circle) or not (e.g., rectangular or ellipse), and whether the drawing is to be done in two or three dimensions. More fine-grained analyses are needed to investigate starting position in the drawing of human faces by right vs. left hand users varying in their preferred writing direction. To separate out visual-attentional from motoric inflences on drawing directionality, drawing tasks could be studied under conditions where visual feedback is available vs. not available (see Chokron et al., 2004). Finally, more studies should use within-subjects designs to examine the degree of consistency in directionality effects across production, perception, preference, and memory tasks.

A number of studies have accumulated demonstrating the influence of reading/writing habits on performance on spatial tasks used in studies of hemispheric asymmetry. Directional biases have also been suggested to underlie other domains as well, such as representational momentum (Halpern \& Kelly, 1993; Spalek \& Hammad, 2005), line bisection (Zivotovsky, 2004), and the perception of numerical magnitude (Shaki, Fischer, \& Petrusic, 
2009). In many of these cases the interactive effects of script directionrelated motoric habits and tensor/flexor-related hand movement biases have not been sufficiently tested. What is sufficiently evident, though, is that motor imagery affects the way we interact with objects in perception, production, and memory and, thus, that the variable of hand movement needs to be foregrounded in models of spatial cognition for its implications for embodiment approaches to cognition (e.g., Fischer \& Zwaan, 2008).

\section{References}

Adamson, Maheen \& Joseph Hellige (2006). Hemispheric differences for identification of words and nonwords in Urdu-English bilinguals. Neuropsychology, 20, 232-248.

Alter, Isabelle (1989). A cerebral origin for “directionality”. Neuropsychologia, 27, 563-573.

Alter, Isabelle, Stephanie Rein \& Alfredo Toro (1989). A directional bias for studies of laterality. Neuropsychologia, 27, 251-257.

Banich, Marie, Wendy Heller \& Jerre Levy (1989). Aesthetic preference and picture asymmetries. Cortex, 25, 187-195.

Bartolomeo, Paolo (2007). Visual neglect. Current Opinion in Neurology, 20, 381386.

Beaumont, J. Graham (1985). Lateral organization and aesthetic preference: The importance of peripheral visual asymmetries. Neuropsychologia, 23, 103-113.

Braswell, Gregory S. \& Karl S. Rosengren (2002). The role of handedness in graphic production: Interactions between biomechanical and cognitive factors in drawing development. British Journal of Developmental Psychology, 20, 581-600.

Brown, Judson S., K.B. Knauft \& G. Rosenbaum (1948). The accuracy of positioning movements as a function of their direction and extent. American Journal of Psychology, 61, 167-182.

Chokron, Sylvie (2002). On the origin of free-viewing perceptual asymmetries. Cortex, 38, 109-112.

Chokron, Sylvie, Pascale Colliot \& Paolo Bartolomeo (2004). The role of vision in spatial representation. Cortex, 40, 281-290.

Chokron, Sylvie \& Maria De Agostini (2000). Reading habits influence aesthetic preference. Cognitive Brain Research, 10, 45-49.

Crovitz, Herbert F. (1962). On direction in drawing a person. Journal of Consulting Psychology, 26, 196.

deAgostini, Maria \& Sylvie Chokron (2002). The influence of handedness on profile and line drawing directionality in children, young, and older normal adults. Brain and Cognition, 48(2-3), 333-336.

De'Sperati, C. \& N. Stucchi (1997). Recognizing the motion of a graspable object is guided by handedness. NeuroReport, 8, 2761-2765. 
Dickinson, C. \& H. Intaub (2009). Spatial asymmetries in viewing and remembering scenes: Consequences of an attentional bias? Attention, Perception \& Psychophysics, 71, 1251-1262.

Dobel, Christian, Gil Diesendruck \& Jens Bolte (2007). How writing system and age influence spatial representations of actions. Psychological Science, 18(6), 487-491.

Dreman, S.B. (1974). Directionality trends as a function of handedness and of reading and writing habits. American Psychologist, 37, 247-254.

Eviatar, Zohar (1997). Language experience and right hemisphere tasks: The effects of scanning habits and multilingualism. Brain and Language, 58, 157-173.

Farias, D., C. Davis \& G. Harrington (2006). Drawing: Its contribution to naming in aphasia. Brain and Language, 97, 53-63.

Fischer, Martin \& Rolf Zwaan (2008). Embodied language: A review of the role of the motor system in language comprehension. The Quarterly Journal of Experimental Psychology, 61, 825-850.

Freimuth, Marilyn \& Seymour Wapner (1979). The influence of lateral organization on the evaluation of paintings. British Journal of Psychology, 70, 211-218.

Gaffron, M. (1950). Left and right in pictures. Art Quarterly, 13, 312-331.

Glenn, S.M. (1995). Handedness and the development of direction and sequencing in children's drawings of people. Educational Psychology, 15, 11-21.

Gross, Charles G. \& Marc H. Bornstein (1978). Left and right in science and art. Leonardo, 11, 29-38.

Halpern, Andrea \& Michael Kelly (1993). Memory biases in left vs. right implied motion. Journal of Experimental Psychology: Learning, Memory and Cognition, 19, 471-484.

Heath, Robin L., Oula Mahmasanni, Aida Rouhana, Nader Nassif (2005). Comparison of aesthetic preferences among Roman and Arabic script readers. Laterality, 10, 399-411.

Heath, Robin L., Aida Rouhana \& Dana Abi Ghanem (2005). Asymmetric bias in perception of facial affect among Roman and Arabic script readers. Laterality, $10,52-64$.

Hernandez, N., A. Metzger, R. Magne, F. Bonnet-Brilhault, S. Roux, C. Barthelmy \& J. Martineau (2009). Exploration of core features of a human face by healthy and autistic adults analyzed by visual scanning. Neuropsychologia, 47, 1004-1012.

Heron, Woody (1957). Perception as a function of retinal locus and attention. American Journal of Psychology, 70, 38-48.

Hsiao, Janet H. \& Garrison Cottrell (2009). Two fixations suffice in face recognition. Psychological Science, 19, 998-1006.

Jensen, B. (1952). Left-right orientation in profile drawing. Perceptual and Motor Skills, 65, 80-83.

Karev, George (1999). Directionality in right, mixed and left handers. Cortex, 35 , 423-431.

Kinsbourne, Marcel (1970). The cerebral basis of lateral asymmetries in attention. Acta Psychologica, 33, 193-201. 
Lehman, Elyse B. \& Jacqueline Goodnow (1975). Directionality in copying: Memory, handedness, and alignment effects. Perceptual and Motor Skills, 41, 863-872.

Levy, Jerre (1976). Lateral dominance and aesthetic preference. Neuropsychologia, $14,431-445$.

Levy, Jerre, Wendy Heller, Marie T. Banich \& L. Burton (1983). Asymmetry of perception in free viewing of chimeric faces. Brain and Cognition, 2, 404-419.

Levy, Jerre \& M. Reid (1978). Variations in cerebral organization as a function of handedness, hand posture in writing, and sex. Journal of Experimental Psychology: General, 107, 119-144.

Maass, Anne \& A. Russo (2003). Directional bias in the mental representation of spatial events: Nature or culture? Psychological Science, 14, 296-301.

Martin, Marian \& G. Jones (1999). Motor imagery theory of a contralateral handedness effect in recognition memory: Toward a chiral psychology of cognition. Journal of Experimental Psychology: General, 128, 265-282.

Mead, Andrew M. \& John P. McLaughlin (1992). The roles of handedness and stimulus asymmetry in aesthetic preference. Brain and Cognition, 20,300-307.

McKelvie, Stuart (2001). Is memory for head orientation based on a left-looking schema? The Journal of General Psychology, 12, 209-225.

McLaughlin, John P. \& Kimberly E. Murphy (1992). Preference for profile orientation in portraits. Empirical Studies of the Arts, 12, 1-7.

McLaughlin, John (1986). Aesthetic preference and lateral preferences. Neuropsychologia, 24, 587-590.

Nachshon, Israel (1981). Cross-cultural differences in directionality. International Journal of Psychology, 16, 199-211.

Nachshon, Israel (1983). Directional preferences of bilingual children. Perceptual and Motor Skills, 56, 747-750.

Nachson, Israel, Einat Argaman \& Assaf Luria (1999). Effects of directional habits and handedness on aesthetic preference for left and right profiles. Journal of Cross Cultural Psychology, 30, 106-114.

Nazir, Tatjana A., Nadia Ben-Boutayab, Nathalie Decoppet, Avital Deutsch, Ram Frost (2004). Reading habits, perceptual learning, and recognition of printed words. Brain \& Language, 88, 294-312.

Padakannaya, Prakash, M.L. Devi, B. Zaveria, Shyamala K. Chengappa \& Jyotsna Vaid (2002). Directional scanning effect and strength of reading habit in picture naming and recall. Brain and Cognition, 48, 484-490.

Palmer, Stephen, Jonathan Gardner \& Thomas Wickens (2008). Aesthetic issues in spatial composition: effects of position and direction on framing single objects. Spatial Vision, 21, 421-449.

Reed, G. \& A.C. Smith (1961). Laterality and directional preference in a simple perceptual motor task. Quarterly Journal of Experimental Psychology, 13, $122-124$.

Sakhuja, Tripti, Gyan C. Gupta, Maharaj Singh \& Jyotsna Vaid (1996). Reading habits affect asymmetries in facial affect judgments: A replication. Brain and Cognition, 32, 162-165. 
Scheirs, J.G. (1990). Relationships between the direction of movements and handedness in children. Neuropsychologia, 28(7), 743-748.

Shaki, Samuel, Michael Fischer \& William Petrusic (2009). Reading habits for both words and numbers contribute to the SNARC effect. Psychonomic Bulletin \& Review, 16, 328-331.

Singh, Maharaj, Jyotsna Vaid \& Tripti Sakhuja (2000). Reading/writing vs. handedness influences on line length estimation. Brain and Cognition, 43(1-3), 398-402.

Spalek, Thomas \& Sherief Hammad (2005). The left-to-right bias in inhibition of return is due to the direction of reading. Psychological Science, 16(1), 15-18.

Symes, Ed, Rob Ellis \& Michael Tucker (2007). Visual object affordances: Object orientation. Acta Psychologica, 124, 238-255.

Taguchi, Masanori \& Yutaka Noma (2005). Relationships between directionality and orientation in drawings by young children and adults. Perceptual \& Motor Skills, 101(1), 90-94.

Takala, Martti (1951). Asymmetries of the visual space, 1-175, Helsinki.

Trojano, Luigi, Dario Grossi \& Tamar Flash (2009). Cognitive neuroscience of drawing: Contributions of neuropsychological, experimental and neurofunctional studies. Cortex, 45, 269-277.

Vaid, Jyotsna (1988). Asymmetries in tachistoscopic word recognition: Scanning effects re-examined. International Journal of Neuroscience, 253-258.

Vaid, Jyotsna (1995). Script directionality affects nonlinguistic performance: Evidence from Hindi and Urdu. In Insup Taylor and David Olson (Eds.), Scripts and literacy (pp. 295-310). Kluwer.

Vaid, Jyotsna (1998). Cultural vs. biomechanical influences on a graphic production task. Brain and Cognition, 37(1), 75-78.

Vaid, Jyotsna \& Hsin-Chin Chen (2009). Drawing directionality as a function of handedness, hand used and script directionality. Unpublished manuscript, Texas A\&M University.

Vaid, Jyotsna, Chaitra Rao \& Hsin-Chin Chen (2009). Right visual advantage characterizes lexical decision in rightward and leftward scripts: Evidence from Hindi/Urdu. Unpublished manuscript, Texas A\&M University.

Vaid, Jyotsna \& Maharaj Singh (1989). Asymmetries in the perception of facial affect: Is there an influence of reading habits? Neuropsychologia, 27, 1277-1287

Vaid, Jyotsna, Maharaj Singh, Tripti Sakhuja \& Gyan C. Gupta (2002). Stroke direction asymmetry in figure drawing: Influence of handedness and reading/writing habits. Brain and Cognition, 597-602.

Viggiano, Maria P. \& Manila Vannucci (2002). Drawing and identifying objects in relation to semantic category and handedness. Neuropsychologia, 40, 14821487.

Van Sommers, Peter (1984). Drawing and cognition: Descriptive and experimental studies of graphic production processes. Cambridge: Cambridge University Press.

Zivotofsky, Ari Z. (2004). Choosing sides: lateralization in line trisection and quadrisection as a function of reading direction and handedness. Cognitive Brain Research, 20, 206-211. 